\title{
Tumor adenomatoide de tubas uterinas, reporte de un caso y revisión de la literatura.
}

Jina Paola Burbano Obando, Médico patólogo del Laboratorio Citosalud, Universidad del Cauca.: jinaburbano@hotmail.com

Harold Jofre Bolaños Bravo, Médico patólogo y docente del departamento de patología, Universidad del Cauca; $\mathrm{Mg}$. En Oncología Molecular, Centro de Estudios Biosanitarios.

Fecha de recepción: 05/06/2018

Fecha de corrección: 11/01/2019

Fecha de aprobación 04/04/2019

Fecha de publicación: 30/06/2019

\section{Resumen}

Los tumores adenomatoides del tracto genital son neoplasias poco frecuentes, de comportamiento benigno; debido a sus características histológicas se confunden fácilmente con tumores de estirpe vascular. Estudios recientes demuestran que los tumores adenomatoides del tracto genital femenino están genéticamente definidos por la mutación TRAF7 que activa la expresión aberrante de la vía NFkB. Presentamos el caso de una mujer de 27 años, asintomática, a quien se le realiza salpingectomía bilateral como método de anticoncepción definitivo; en el examen macroscópico de la tuba derecha se encuentra formación quística que por sus características microscópicas es diagnosticada como tumor adenomatoide de la tuba uterina. Finalmente se realiza una revisión de la literatura.

Palabras claves: Tumor adenomatoide, neoplasias benignas de la tuba uterina, factor nuclear kappa B, TRAF7, inmunohistoquímica.

Copyright (C) Facultad de Ciencias de la Salud de la Universidad Tecnológica de Pereira. 1995-2018. Todos los derechos reservados.
Adenomatoid uterine tube tumor, case report and literature review

\begin{abstract}
The adenomatoid tumors of the genital tract are rare neoplasms, with benign behavior; due to their histological characteristics, they are easily confused with tumors of vascular origin. Recent studies show that adenomatoid tumors of the female genital tract are genetically defined by the TRAF7 mutation that activates the aberrant expression of the NFkB pathway. We present the case of a 27-year-old woman, asymptomatic, who underwent bilateral salpingectomy as a method of definitive contraception; In the macroscopic examination of the right tuba, cystic formation is found which, due to its microscopic characteristics, is diagnosed as an adenomatoid tumor of the uterine tube. Finally, a review of the literature is made.
\end{abstract}

Key words: Adenomatoid tumor, benign neoplasms of the uterine tuba, nuclear factor kappa B, TRAF7, immunohistochemistry.

Copyright (C) Facultad de Ciencias de la Salud de la Universidad Tecnológica de Pereira. 1995-2018. Todos los derechos reservados.

\section{Introducción}

Los tumores de las trompas uterinas son raros pero histológicamente se reconocen los mismos subtipos que en ovario; los tumores adenomatoides hacen parte de los tumores benignos más frecuentes y por lo general su hallazgo es incidental $(1,2)$. La falta de claridad respecto a su origen, explica por qué ha recibido diferentes denominaciones como: linfangioma, adenomioma, reticuloendotelioma, tumor angiomatoide, adenofibroma, adenoma o mesotelioma, pero solo hasta el año de 1945 Golden y colaboradores propusieron el término de tumor adenomatoide, que actualmente es aceptado(3, 4). Con la aplicación de diferentes pruebas se ha comprobado su estirpe mesotelial (4).

\section{Caso clínico}

Una mujer de 27 años con paridad satisfecha, sin antecedentes médicos de relevancia y asintomática, es llevada a salpingectomía como método definitivo de planificación; se envían los fragmentos de las trompas para estudio histopatológico, al examen macroscópico la tuba uterina derecha que medía $7.8 \mathrm{x} 1 \mathrm{~cm}$ de longitud, a $0.2 \mathrm{~cm}$ de la fimbria presenta una formación nodular al parecer dependiente de la serosa, al corte se trata de una masa redonda con dimensiones de $0,5 \times 0,5 \mathrm{~cm}$, al corte es bien circunscrita, pero no encapsulada, blanquecina homogénea y elástica; en el análisis histopatológico se identifica tuba uterina con engrosamiento de la mucosa y la muscular a expensas de células ligeramente pleomorfas, aplanadas o cuboidales, de núcleos redondos con cromatina laxa y citoplasma eosinófilo que se disponen formando estructuras muy similares a vasos, algunas de esas luces son atravesadas por delgadas bandas eosinófilas, no se identifican zonas de necrosis y se cuenta 1 mitosis por 10 campos de alto poder; ante la sospecha de neoplasia de estirpe vascular se solicitan marcadores de inmunohistoquimica, expresando positividad para calretinina y vimentina, en cambio anticuerpos como CD34, actina de musculo liso y citoqueratina 5/6 no mostraron reactividad, el índice de proliferación celular (ki67) fue menor al 10\%. (Ver figura 1)

\section{DISCUSIÓN}

Los tumores adenomatoides son neoplasias benignas, propias del tracto genital masculino o femenino(5) siendo más frecuentes en los primeros(3, 6 ), se han descrito ubicaciones extragenitales menos comunes como las glándulas adrenales, apéndice, corazón, hígado, saco herniario, mesenterio intestinal, ganglios linfáticos, mediastino, omento, páncreas, peritoneo y ombligo $(7,8)$.

El miometrio es el lugar en donde más frecuentemente han sido reportados y con un patrón infiltrativo, para el caso del tracto genital femenino, seguido por las trompas uterinas $(5,7)$. Macroscópicamente se trata de nódulos pequeños bien circunscritos, de color gris blanquecino, muy raramente bilaterales con un tamaño máximo de $2.7 \mathrm{~cm}(4,5)$. Generalmente el tumor adenomatoide es asintomático, de hallazgo incidental, en donde la cirugía se realiza bajo otras indicaciones como: endometriosis, adenomiosis, leiomiomatoisis, hiperplasia endometrial, pólipos endocervicales, 
neoplasia cervical intraepitelial (NIC III), quiste de cuerpo lúteo y quiste paraovarico (9).

La histogénesis de los tumores adenomatoides ha sido controversial a través de los tiempos proponiéndose el origen endotelial, mesonefrico, mesotelial y mulleriano, no obstante en general se ha aceptado la estirpe mesotelial, mediante varios marcadores de inmunohistoquímica, estudio de microscopia electrónica e histoquímica $(4,8,10)$, inclusive se ha demostrado continuidad de las células de revestimiento peritoneal y las células que tapizan las estructuras similares a canales o túbulos(11).

La patogénesis no es bien conocida, aunque se plantea disfunción en la señalización de las citoquinas entre las células endoteliales luego de estudios como el de Mizutani y colaboradores (2016) en donde encontraron que el trasplante renal, la inmunosupresión secundaria y el tiempo de diálisis son factores de riesgo para desarrollar estas neoplasias(12); por otro lado se ha comprobado una activación aberrante de la vía del factor nuclear kappa $\mathrm{B}(\mathrm{NF}-\mathrm{kB})$ en tumores adenomatoides del tracto genital femenino y masculino, cuyo perfil genético es portador de mutaciones somáticas sin sentido en el gen TRAF7 que codifica una E3 ubiquitin ligasa perteneciente a la familia de factores asociados al receptor del factor de necrosis tumoral (TRAF). In vitro hay aumento de la fosforilación de NF-kB y mayor expresión de la molécula de adhesión celular L1 (L1CAM), que es un marcador de la activación de ésta vía; lo que concuerda con el perfil de inmunohistoquímica (13).

Histológicamente hay diferentes patrones como el adenoide, angiomatoide, quístico, glandular, sólido, oncocito, isquémico reactivo y tubular (7). Tanto en hombres como en mujeres se trata de neoplasias bien circunscritas más no encapsuladas, de aspecto glandular o acinar tapizados por células planas, cuboidales o muy raramente columnares, de citoplasma ligeramente eosinófilo, vacuolado, núcleos pequeños y nucléolos inconspicuo; la unión de proyecciones citoplasmáticas, que contienen material mitocondrial o tonofilamentos escasos, de células vecinas forman los patognomónicos filamentos que cruzan los espacios pseudotubulares y son típicos de estos tumores (en el $100 \%$ ), dichos filamentos pueden ser tan delgados que se asimilan a los adipocitos, sobre los lúmenes que contienen material basófilo mucicarmin y PAS positivo son menos visibles dichos filamentos, el estroma subyacente es fibroso o denso, con algunos focos de hialinización (5), las mitosis son raras(4); otra característica descrita son los agregados linfoides que son frecuentes en los tumores del tracto genital masculino, diferente a los femeninos que se encuentran hasta en el $50 \%$ de los casos, una explicación razonable es la mayor exposición de los primeros a trauma (7) dada la variedad de patrones histológicos posibles del tumor adenomatoide, son amplios los diagnósticos diferenciales que deben considerarse, varios de ellos de naturaleza maligna, sin embargo se recomienda la evaluación cuidadosa de las características morfológicas y el uso de marcadores de inmunohistoquímica si la duda persiste (8). El inmunofenotipo de los tumores adenomatoides del tracto genital femenino es peculiar y varios de los marcadores confirman la histogénesis mesotelial; reacciona con anticuerpos como CKAE1/CAM5.2, D2-40 y calretinina en todos los casos, WT-1 reactivo en la mayoría (97\%) y ocasionalmente es reactivo para caldesmon y CK5/6 (3\% y $16 \%$ repectivamente) $(7,9)$; en cambio no reacciona con marcadores como ER, AR, CD31, CD34 y PAX8, el índice de proliferación ki67 menor o igual al 10\% reafirma su benignidad(9). Otros marcadores expresados por estos tumores son vimentina, HBME-1 y OC125, los dos últimos son antígenos asociados al mesotelio (14). Confirmando la activación errada del NF$\mathrm{kB}$ según la fenotipificación ya expuesta, los tumores adenomatoides expresan reactividad contra L1CAM diferente al mesotelio normal, mesoteliomas peritoneales malignos y los quistes peritoneales de inclusión multiloculares que no fueron reactivos (13).

\section{Agradecimientos:}

Al Departamento de patología de la Universidad del Cauca y a sus docentes por el apoyo en la interpretación y análisis del caso; además de a la Compañía de Patólogos del Cauca por proporcionar los cortes histológicos, la hematoxilina y eosina y los anticuerpos de inmunohistoquímica aquí presentados.
Conflictos de interés: Los autores declaramos que no tenemos conflictos de interés

\section{Referencias}

1. Alvarado-Cabrreo I, Cheung A, Caduff R. Tumours of the fallopian tube. WHO Classification of Tumours of Female Reproductive Organs. 2014.

2. Fletcher CD. Diagnostic histopathology of tumors: 1-volume Elsevier Health Sciences; 2013.

3. Youngs LA, Taylor HB. Adenomatoid tumors of the uterus and fallopian tube. American journal of clinical pathology. 1967;48(6):537-45.

4. Maryann BM, Umakanthan S, Rai S, Rao A. Adenomatoid tumor of the fallopian tube-A rare case report and review of literature. International Journal of Medical and Applied Sciences. 2015;4(Sup 1):4-7.

5. Hes O, Perez-Montiel DM, Cabrero IA, Zamecnik M, Podhola M, Sulc M, et al. Thread-like bridging strands: a morphologic feature present in all adenomatoid tumors. Annals of diagnostic pathology. 2003;7(5):273-7.

6. Hsu Y-H. Adenomatoid tumor of the uterus. Tzu Chi Medical Journal. 2012;24(1):30-1.

7. Sangoi AR, McKenney JK, Schwartz EJ, Rouse RV, Longacre TA. Adenomatoid tumors of the female and male genital tracts: a clinicopathological and immunohistochemical study of 44 cases. Modern Pathology. 2009;22(9):1228.

8. Sheng B, Zhang Y-P, Wei H-H, Ma M, Nan X. Primary adenomatoid tumor of the testis: report of a case and review of literature. International journal of clinical and experimental pathology. 2015;8(5):5914.

9. Wachter DL, Wünsch PH, Hartmann A, Agaimy A. Adenomatoid tumors of the female and male genital tract. A comparative clinicopathologic and immunohistochemical analysis of 47 cases emphasizing their site-specific morphologic diversity. Virchows archiv. 2011;458(5):593-602.

10. Klintorp S, Grinsted L, Franzmann M-B. Adenomatoid tumor of the uterus. European Journal of Obstetrics \& Gynecology and Reproductive Biology. 1993;50(3):255-7.

11. Sağlican Y, Kurtulmus N, Tunca F, Süleyman E. Mesothelial derived adenomatoid tumour in a location devoid of mesothelium: adrenal adenomatoid tumour. BMJ case reports. 2015;2015:bcr2015211147.

12. Mizutani T, Yamamuro O, Kato N, Hayashi K, Chaya J, Goto N, et al. Renal transplantation-related risk factors for the development of uterine adenomatoid tumors. Gynecologic oncology reports. 2016;17:96.

13. Goode B, Joseph NM, Stevers M, Van Ziffle J, Onodera C, Talevich E, et al. Adenomatoid tumors of the male and female genital tract are defined by TRAF7 mutations that drive aberrant NF-kB pathway activation. Modern Pathology. 2017.

14. Delahunt B, Eble J, King D, Bethwaite P, Nacey J, Thornton A. Immunohistochemical evidence for mesothelial origin of paratesticular adenomatoid tumour. Histopathology. 2000;36(2):109-15. 